\title{
14-3-3 zeta protein secreted by tumor associated monocytes/ macrophages from ascites of epithelial ovarian cancer patients
}

\author{
Ryuji Kobayashi - Michael Deavers · Rebecca Patenia • \\ Travis Rice-Stitt $\cdot$ Jeremy Halbe $\cdot$ Stacie Gallardo . \\ Ralph S. Freedman
}

Received: 21 April 2008/Accepted: 11 June 2008/Published online: 11 July 2008

(C) The Author(s) 2008

\begin{abstract}
Tumor associated monocytes/macrophages (MO/MA) are known contributors to the immune-inflammatory cell environment of advanced epithelial ovarian carcinoma (EOC). The secreted proteome of ascitic MO/ MA was examined as an aid to the discovery of novel proteins in EOC that are likely to have biological relevance in the inflammatory pathways of EOC. Ascitic fluid MO/ MA were isolated from EOC patients, grown short-term in serum-free media. MO/MA supernatants were analyzed for secreted proteins by HPLC fractionation followed by LCtandem mass spectrometric analysis. The 14-3-3 zeta adaptor protein was identified in supernatants of three of three EOC patients but not in supernatants of buffy coat monocytes isolated from normal donors or the established monocyte cell line THP1. Moreover, 14-3-3 zeta was identified in ascitic fluids in eight of eight chemotherapynaïve patients by both immunoblot and mass spectrometric analysis. Immunofluorescent staining for 14-3-3 zeta demonstrated expression of the protein on ascitic and
\end{abstract}

Electronic supplementary material The online version of this article (doi:10.1007/s00262-008-0549-7) contains supplementary material, which is available to authorized users.

R. Kobayashi $(\bowtie) \cdot$ T. Rice-Stitt · J. Halbe

Department of Molecular Pathology, Unit 951, The University of Texas M. D. Anderson Cancer Center, Houston, Texas, US

e-mail: rkobayas@mdanderson.org

\section{Deavers}

Department of Pathology, Unit 85, The University

of Texas M. D. Anderson Cancer Center, Houston, Texas, US

R. Patenia · S. Gallardo · R. S. Freedman $(\bowtie)$

Department of Gynecologic Oncology, Unit 1362,

The University of Texas M. D. Anderson Cancer Center,

1515 Holcombe Blvd., Houston, Texas 77030, US

e-mail: rfreedma@mdanderson.org peritumoral macrophages in EOC patients. 14-3-3 zeta was also expressed on endothelial cells in the peritumoral stroma and partially on tumor cells. Uptake of 14-3-3 zeta was observed in EOC cell lines co-cultured with the recombinant protein expressed in E. coli. It is demonstrated for the first time that the important adaptor protein 14-3-3 zeta is common to the secretome of ascitic MO/MA and the ascites of advanced EOC patients.

Keywords Ovarian cancer - Monocytes/Macrophages · Inflammation $\cdot$ Secreted proteins $\cdot 14-3-3$ zeta

\section{Introduction}

As currently practiced, the treatment of stage III or IV epithelial ovarian carcinoma (EOC) results in a 5-year survival rate of only $25-30 \%$. This poor outcome contrasts with a 10-year survival rate of $90 \%$ for stage I patients, where peritoneal and serosal tumor involvement is absent. Clearly, morbidity and mortality in EOC are related to peritoneal and serosal tumor involvement. The peritoneum protects the integrity of intra-abdominal organs and can also facilitate infiltration of inflammatory cells to sites of infection or injury, thus localizing pathologic effects. Examination of the peritoneum and ascites of patients with advanced EOC reveals high numbers of inflammatory cells comprised largely of monocytes/macrophages (MO/MA) that express markers of activation [13]. The importance of $\mathrm{MO} / \mathrm{MA}$ in disease progression of EOC is supported by several studies [1, 6] and as reviewed previously [7]. For example, elevated blood levels of the MA-differentiating cytokine macrophage colony-stimulating factor 1 (MCSF), which is also produced by MO/MA, correlates both with disease progression and with poor survival [1]. Further, the 
proangiogenic factors interleukin-8 (IL8) and vascular endothelial growth factor (VEGF), which are produced by peritoneal and ascitic MO/MA and by EOC tumor cells and stromal cells, have been shown to correlate with poor survival in EOC patients [6]. Most of the proteins with potential protumor activity which are secreted by tumor associated MO/MA have been identified using antibody based methods. There is also a need to identify other lesser known proteins which may have an important role in tumor progression and metastasis.

The MO derived THP1 cell line was employed as a first step in the examination of the secretome of MO/MA. This was followed by an examination of the secretome of purified MO/MA isolated from the ascitic fluid of EOC patients. Supernatants from buffy coat derived MO/MA were compared to those of ascitic MO/MA. A single protein, 14-3-3 zeta, was identified in each of three supernatants of EOC ascitic MO/MA and also detected in ascites of all eight patients examined. An antibody specific to this protein was generated and costaining experiments demonstrated 14-3-3 zeta expression on ascitic MO/MA, the peritoneum, tumor stroma, endothelial cells, and variably on tumor cells.

\section{Materials and methods}

Isolation of MO/MA from ascitic fluid and collection of supernatant

Human tissues were obtained under University of Texas MD Anderson Cancer Center IRB approved protocol, and written informed consent obtained from all patients. Ascitic $\mathrm{MO} /$ MA from EOC patients were isolated as previously described $[5,13]$. We used a sequential procedure that involved a Ficoll-Hypaque density cushion followed by adherence for $1 \mathrm{~h}$. In the CD163 MO/MA subset experiment a MO Isolation Kit and a MACS separator (Miltenyi Biotec, Auburn, CA) were utilized according to the manufacturer's instructions. Cells were labeled with a hapten-antibody cocktail (containing monoclonal hapten-conjugated CD3, CD7, CD19, CD45RA, CD56 and anti-IgE antibodies) and MACS Anti-Hapten Microbeads, washed with buffer (PBS with $0.5 \%$ human albumin and $1 \mathrm{mM}$ EDTA), and then run through a column placed in a MACS magnetic separator. The unattached MO/MA were collected, while the magnetically labeled $\mathrm{T}$ cells, B cells, NK cells, DC, and basophils remained attached to the column. When large numbers of tumor cells were present, anti-HEA (EpCAM) microbeads were added to the column. Purified MO/MA were then placed in a 6-well plate and cultured in KrebsHensleit buffer. Supernatants were collected after 2-9 days of incubation, based on optimum viability, and in CD163 experiments at 0,24 , and $48 \mathrm{~h}$.

Oligofractionation of secreted proteins from the ascitic $\mathrm{MO} / \mathrm{MA}$ of EOC patients

For secreted proteome analysis, $200 \mu$ l of supernatant was taken from each sample and fractionated by HPLC (Hewlett Packard 1090) using a reversed phase column (Vydac $\mathrm{C} 4,1 \times 250 \mathrm{~mm}$ ). After extensively washing the column to eliminate salts and other small molecules mostly derived from media, the proteins were eluted with an increasing gradient of acetonitrile and collected into three $10 \mathrm{~min}$ fractions. The HPLC gradient was set as follows: $10 \%$ solvent B (70\% acetonitrile/water, $0.091 \%$ TFA), $90 \%$ solvent A (2\% acetonitrile/water, $0.1 \%$ TFA) from 0 $30 \mathrm{~min} ; 50 \% \mathrm{~B}, 50 \% \mathrm{~A}$ at $50 \mathrm{~min} ; 90 \% \mathrm{~B}, 10 \% \mathrm{~A}$ at $60 \mathrm{~min}$ and $90 \% \mathrm{~B}, 10 \% \mathrm{~A}$ until $70 \mathrm{~min}$. After trypsin digestion, the three fractions were subjected to mass spectrometric analysis. This HPLC separation achieves the cleanest sample possible, while still permitting high sensitivity mass spectrometric analysis.

Mass spectrometric analysis

LC-tandem mass spectrometric (LC-MS/MS) analysis of the secreted proteome was performed using an ion-trap mass spectrometer (Thermo Electron LTQ) connected with a reversed phase column (LC-Packings C18 PepMap, $75 \mu \mathrm{m} \times 150 \mathrm{~mm})$. Mascot was used to search the mass spectra against NCBI databases. Proteins that were matched with reasonable mass spectra are listed (Table 1).

Anti-14-3-3 zeta antibodies

Since no commercial antibody specific to $14-3-3$ zeta was available, due to its high homology with other isoforms, a synthetic peptide was designed based on the sequence analysis of all seven 14-3-3 isoforms (Fig. 1) and the crystal structure of 14-3-3 zeta [8]. A peptide with $\mathrm{N}$ terminal cystein was synthesized (CEKFLIPNASQAE, residues 102-113), conjugated to $\mathrm{KLH}$, and used to immunize rabbits. Anti-peptide antibodies were purified by affinity chromatography over a peptide column (GenScript, Piscataway, NJ).

Detection of secreted 14-3-3 zeta in ascitic fluid

Ten microliters of ascitic fluid isolated from patients with ovarian cancer was first cleaned by immunodepletion column chromatography to remove serum albumin and 5 other abundant proteins (MARS, Agilent technologies, Palo Alto, 
Table 1 Secreted proteomes of MO/MA isolated from the ascites of patients with EOC

Buffy coat MCSF (+) (49 proteins)

enolase 1 variant

beta-actin

phosphoglycerate kinas 1

profilin 1

L-plastin

aldolase A

peptidylprolyl isomerase $A$

transketolase

histone $\mathrm{H} 1$

ubiquitin

glutathione transferase

triosephosphate isomerase

S100 calcium-binding protein A9

S100 calcium-binding protein A8

$\mathrm{SH} 3$ domain binding glutamic acid-rich protein like $3-344^{*}$

filamin 1

histone $\mathrm{H} 1 \mathrm{~b}$

glucose phosphate isomerase

transgelin 2

coronin-like protein

vinculin isoform $\mathrm{VCL}$

cystatin A

gelsolin

leukotriene A4 hydrolase

calcium-calmodulin $\mathrm{n}$-terminal domain

histone $\mathrm{H} 2 \mathrm{~B}$

peroxiredoxin 1

HIST1H4F

nonmuscle myosin heavy chain

coactosin-like 1

eukaryotic translation elongation factor 1

$\mathrm{S} 100$ calcium-binding protein A11

neuropolypeptide h3

adenylyl cyclase-associated protein

alpha-1-antitrypsin

Ras suppressor protein 1

pyruvate kinase

adenylate kinase 2

placental protein 23

transaldolase 1

EF hand domain family, member D2

lysozyme

APEX nuclease

heterogeneous nuclear ribonucleoprotein $\mathrm{L}$

thymosin

ATP synthase

macrophage migration inhibitory factor

NADP-dependent isocitrate dehydrogenase

heterogeneous nuclear ribonucleoprotein $M$
Score Peptides |Patient\#290 Day 9 MCSF (+) (5 2 proteins)

apolipoprotein E

L-plastin

alpha-1-antitrypsin

enolase 1 variant

vimentin

beta-actin

aldolase $\mathrm{A}$

pyruvate kinase

chitinase 3-like 1

transketolase

profilin 1

S100 calcium-binding protein $\mathrm{A} 9$

peptidylprolyl isomerase $A$, isoform 1

phosphoglycerate kinas 1

S100 calcium-binding protein A8

glyceraldehyde-3-phosphate dehydrogenase

triosephosphate isomerase 1

NADP-dependent isocitrate dehydrogenase

14-3-3 beta

CD14 antigen precursor

Chain $A$, synthetic ubiquitin with fluoro-leu at $50 \& 67$ histone $\mathrm{H} 2 \mathrm{~B}$

beta-2 microglobulin

S100 calcium binding protein A11

SH3 domain binding glutamic acid-rich protein like 3

matrix metalloproteinase 9 preproprotein

14-3-3 zeta (YWHAZ protein)

crystatin A

brain abundant, membrane attached signal protein 1

leukotriene A4 hydrolase

serum albumin

HIST1H4F

alpha-actin

fructose-1,6-bisphosphatase 1

lactate dehydrogenase $A$ variant

glutathione transferase

cystatin C

thymosin

gelsolin-like capping protein

peroxiredoxin 1

transaldolase 1

tropomyosin 4-anaplastic lymphoma kinase fusion protein

cofilin 1 (non-muscle)

neuropolypeptide h3

proteoglycan 1 , secretory granule

histone H1B

H41 protein

nonmuscle myosin heavy chain

coronin-like protein

heterogeneous nuclear ribonucleoprotein $\mathrm{L}$

lysozyme

$\mathrm{Cu} / \mathrm{Zn}$ superoxide dismutase
CA). One-twentieth of the cleaned ascitic fluid was used for immunoblot analysis with 14-3-3 zeta antibodies (Santa Cruz SC-1019 with greater specificity for zeta and to a lesser extent beta and sigma). The silver stained gel area corresponding to the band in immunoblot analysis was excised and in-gel digested using trypsin. The extracted peptides were analyzed with LC-MS/MS mass spectrometry.

\section{Targeted acquisition in LC-MS/MS}

To increase sensitivity, we selectively acquired $\mathrm{ms} / \mathrm{ms}$ spectra corresponding to the doubly charged states of seven 14-3-3 peptides. Selection of these peptides was based on analyses of digested recombinant material and consideration of the sequence. The mass spectrometer was programmed to sequentially acquire $6 \mathrm{~ms} / \mathrm{ms}$ spectra repeatedly through the run, with $\mathrm{m} / \mathrm{z}$ corresponding to the doubly charged peptides. The isolation width was $4 \mathrm{~m} / \mathrm{z}$ and 2 micro-scans were acquired per spectrum. Normalized collision energy was set to 35 . Activation $\mathrm{Q}$ was 0.250 with an activation time of $30 \mathrm{~ms}$.

\section{Construction of N-terminal FLAG-tagged 14-3-3 zeta} protein

cDNA for 14-3-3 zeta (accession number BC003623) was obtained from Open Biosystems (www.openbiosystems. com). The sequence was inserted into the pT7-FLAG-1 
Table 1 continued

\begin{tabular}{|c|c|c|c|c|c|}
\hline Patient\#287 Day 7 MCSF (+) (32 proteins) & Score & Peptides & Buffy coat MCSF (-) (58 proteins) & Score & Peptides \\
\hline L-plastin & 774 & 15 & enolase 1 variant & 1463 & 23 \\
\hline serum albumin & 569 & 14 & phosphoglycerate kinas 1 & 955 & 18 \\
\hline vimentin & $394 *$ & 10 & transketolase & 919 & 17 \\
\hline S100 Ca-binding protein A9 & 383 & 9 & L-plastin & 773 & 14 \\
\hline triosephosphate isomerase & 372 & 6 & glucose phosphate isomerase & 626 & 10 \\
\hline filamin 1 & $354 *$ & 7 & aldolase $\mathrm{A}$ & 597 & 10 \\
\hline ferritin light chain & 310 & 7 & vinculin isoform VCL & 587 & 12 \\
\hline histone $\mathrm{H} 4$ & 288 & 6 & triosephosphate isomerase 1 & 544 & 9 \\
\hline beta-actin & 241 & 6 & profilin 1 & $457^{*}$ & 5 \\
\hline alpha-1-antitrypsin & 217 & 3 & filamin 1 & $450 *$ & 9 \\
\hline leucine aminopeptidase & 216 & 4 & S100 Ca binding protein A9 & 438 & 9 \\
\hline transketolase & 212 & 3 & beta-actin & $432 *$ & 8 \\
\hline aldolase A & 208 & 4 & histone $\mathrm{H} 1$ & 431 & 8 \\
\hline Ig kappa chain & 195 & 2 & peptidylprolyl isomerase $\mathrm{A}$ & 425 & 7 \\
\hline Ig G1 H Nie & 195 & 4 & alpha-actin & 413 & 12 \\
\hline cystatin A & 192 & 3 & Chain $A$, synthetic ubiquitin with fluoro-leu at $50 \& 67$ & 398 & 8 \\
\hline fibronectin & 191 & 5 & glutathione transferase & 388 & 5 \\
\hline histone $\mathrm{H} 2 \mathrm{~A}$ & 190 & 2 & S100 Ca binding protein $\mathrm{A} 8$ & $379 *$ & 6 \\
\hline haptoglobin & 180 & 4 & leukotriene A4 hydrolase & 353 & 7 \\
\hline MCSF & 174 & 4 & gelsolin & 339 & 6 \\
\hline thymosin & 172 & 3 & coactosin-like 1 & 329 & 7 \\
\hline profilin 1 & 149 & 3 & SH3 domain binding glutamic acid-rich protein like 3 & $306^{*}$ & 5 \\
\hline ubiquitin & 136 & 3 & transgelin 2 & 275 & 4 \\
\hline alpha actin & 124 & 4 & histone $\mathrm{H} 1 \mathrm{~b}$ & $270^{*}$ & 5 \\
\hline thymosin beta 10 & 115 & 2 & alpha-1-antitrypsin & 229 & 3 \\
\hline ATP syntase & 104 & 1 & HIST1H4F & 198 & 4 \\
\hline cystatin C & 97 & 1 & histone $\mathrm{H} 2 \mathrm{~B}$ & 196 & 4 \\
\hline beta-2 microglobulin & 94 & 1 & EF hand domain family, member D2 & 185 & 4 \\
\hline 14-3-3 zeta (YWHAZ protein) & 81 & 1 & adenylyl cyclase-associated protein 1 & 179 & 3 \\
\hline SH3 domain binding glutamic acid-rich protein like 3 & 81 & 1 & peroxiredoxin 1 & 166 & 4 \\
\hline HSP70 & 76 & 2 & $\mathrm{Cu} / \mathrm{Zn}$ superoxide dismutase & 166 & 3 \\
\hline \multirow[t]{3}{*}{$14-3-3$ beta } & 75 & 1 & lysozyme & 163 & 4 \\
\hline & & & coronin-like protein & 162 & 3 \\
\hline & & & placental protein 23 & 162 & 3 \\
\hline \multirow[t]{2}{*}{ Patient\#288 Day 2 MCSF (+) (14 proteins) } & Score & Peptides & transaldolase 1 & 162 & 3 \\
\hline & & & ALB protein & 161 & 3 \\
\hline desmoplakin & 177 & 4 & galactose-specific lectin (galectin-3) & 156 & 3 \\
\hline alpha-actin & 170 & 3 & peroxiredoxin 6 & 155 & 2 \\
\hline heat shock $27 \mathrm{kDa}$ protein 1 & $160 *$ & 1 & phosphoglycerate mutase 1 & 142 & 3 \\
\hline epithelial cell marker protein 1 , stratifin & 143 & 2 & HNRPF protein & 139 & 3 \\
\hline DNA-binding protein $B$ & $141^{*}$ & 1 & phosphoglucomutase 2 & 139 & 3 \\
\hline histone $\mathrm{H} 1$ & $128^{*}$ & 1 & heterogeneous nuclear ribonucleoprotein $\mathrm{L}$ & 139 & 2 \\
\hline glutathione transferase & 124 & 1 & carbonic anhydrase II & 136 & 4 \\
\hline triosephosphate isomerase & 118 & 2 & peroxiredoxin 3 & 130 & 2 \\
\hline 14-3-3 zeta (YWHAZ protein) & 113 & 2 & APEX nuclease & 128 & 3 \\
\hline annexin $\mathrm{A} 1$ & 110 & 1 & adenylate kinase 2 & 126 & 3 \\
\hline SH3 domain binding glutamic acid-rich protein like 3 & 100 & 2 & S100 calcium binding protein A11 & 123 & 2 \\
\hline phosphoglycerate mutase & 78 & 1 & nonmuscle myosin heavy chain & 122 & 2 \\
\hline ubiquitin & 62 & 1 & S100 calcium binding protein A4 & 116 & 3 \\
\hline \multirow[t]{9}{*}{ macrophage migration inhibitory factor } & 62 & 1 & PYD and CARD domain containing isoform a & 114 & 2 \\
\hline & & & S100 calcium binding protein $A 6$ & 105 & 3 \\
\hline & & & eukaryotic translation elongation factor 1 & 89 & 2 \\
\hline & & & NADP-dependent isocitrate dehydrogenase & 85 & 1 \\
\hline & & & migration inhibitory factor & 73 & 1 \\
\hline & & & brain abundant, membrane attached signal protein 1 & 68 & 1 \\
\hline & & & cystatin A & 65 & 1 \\
\hline & & & pyruvate kinase & 61 & 1 \\
\hline & & & thymosin beta 10 & 53 & 1 \\
\hline
\end{tabular}

bacterial expression vector (www.sigmaaldrich.com). Protein was expressed in E. coli BL21 cells, purified with Anti-FLAG Agarose M2 (Sigma-Aldrich, St. Louis, Missouri), and eluted with FLAG peptide $(100 \mu \mathrm{g} / \mathrm{ml})$ in $50 \mathrm{mM}$ Tris- $\mathrm{HCl}$ with $150 \mathrm{mM} \mathrm{NaCl}, \mathrm{pH} 7.4$. The amino acid sequence of the N-terminal through the 14-3-3 zeta start Methionine is "MDYKDDDDKLAAAM" (FLAG epitope sequence is underlined).
Indirect immunofluorescence staining and confocal microscopy

Antibody costaining of ascitic cells, tumor and peritoneum tissues was performed by methods and with reagents that we have described previously utilizing cell localization primary antibodies for cytokeratin (tumor cells), CD163(MO/MA), CD31(endothelial cells), and CD3 (T-cells) [4]. 
Table 1 continued

Patient\# 290 Day 9 MCSF (-) (39 proteins)

\section{vimentin}

enolase 1 variant

L-plastin

phosphoglycerate kinas 1

beta-actin

aldolase $\mathrm{A}$

apolipoprotein $\mathrm{E}$

14-3-3 zeta (YWHAZ protein)

S100 calcium-binding protein A9

triosephosphate isomerase 1

transketolase

gelsolin-like capping protein

chitinase 3 -like 1

alpha-1-antitrypsin

CD14 antigen precursor

leukotriene A4 hydrolase

glyceraldehyde-3-phosphate dehydrogenase

ALB protein

14-3-3 beta

coronin-like protein

$\mathrm{SH} 3$ domain binding glutamic acid-rich protein like 3

tropomyosin 4-anaplastic lymphoma kinase fusion protein

lactate dehydrogenase $A$ variant

pyruvate kinase

fructose-1,6-bisphosphatase 1

NADP-dependent isocitrate dehydrogenase

gelsolin

glutathione transferase

histone $\mathrm{H} 2 \mathrm{~B}$

cystatin $\mathrm{C}$

peroxiredoxin 1

peptidylprolyl isomerase A (PPIA)

brain abundant, membrane attached signal protein 1

thymosin (TMSB4L)

S100 calcium-binding protein A11

phosphoglycerate mutase 1

transgelin 2

histone $\mathrm{H} 1 \mathrm{~b}$

proteoglycan 1 , secretory granule

The peptides identified in the secreted proteome analysis are summarized in different colors. Proteins found in both the control (buffy coat) and patients are in blue. Those unique to control are in green. Proteins found in either patients or control, but with no significant pattern, are coded in black. A protein found in all patients but not in the buffy coat control is coded in red. In mass spectrometry analysis, a higher score indicates higher confidence of identification. The number under the peptide column indicates the number of peptides matched in a sequence database search. $\mathrm{MCSF}(+)$ indicates MO/MA incubated with MCSF, and MCSF $(-)$ indicates those incubated without MCSF. The Mascot scores of proteins identified in multiple HPLC fractions were combined and listed as the total score (indicated with an asterisk)

Established ovarian tumor cell lines CaOV3 and MDA2774, 100,000-50,000 cells per well, were seeded into 8 chamber polystyrene vessel tissue culture treated glass slides (Becton, Franklin Lakes, NJ) and incubated for $24 \mathrm{~h}$ at $5 \% \mathrm{CO}_{2}$ incubator. Cultured cells were washed twice with warmed RPMI 1640 only, then replaced with fresh media without and with FLAG-tagged 14-3-3 zeta in concentration of $2 \mu \mathrm{g} /$ well at 5, 30, 60 and $120 \mathrm{~min}$. After exposure to FLAG-tagged protein, cells were washed three times with $\mathrm{PBS}$ without $\mathrm{Ca}^{++}$and $\mathrm{Mg}^{++}$, fixed immediately with cold $\left(-20^{\circ} \mathrm{C}\right)$ methanol for $5 \mathrm{~min}$ and washed three times with PBS. Permeabilization of cells were applied by using $0.5 \%$ Triton $\mathrm{X}-100$ for $15 \mathrm{~min}$ and nonspecific binding proteins were blocked by adding $5 \%$ normal goat serum for $30 \mathrm{~min}$. The primary antibody, antiFLAG M2 monoclonal antibody IgG1, $20 \mu \mathrm{g} / \mathrm{ml}$ (SigmaAldrich, St. Louis, Missouri) was incubated on fixed cells for overnight at $4^{\circ} \mathrm{C}$ and reacted with $\mathrm{Cy} 2$-conjugated (green) AffiniPure goat anti-mouse IgG, Fcg subclass 1- specific (Jackson ImmunoResearch Laboratories, West Grove, PA). The nuclei of cells were stained with TO-PRO3, 1/2,000 dilution (Molecular Probes, Eugene OR) for $15 \mathrm{~min}$; washed and mounted with Slow-Fade Gold anti-fade reagent (Molecular Probes, Eugene OR). Cells were viewed with an Olympus FV500 laser scanning confocal microscope; images were captured at $200 \times$ magnification and zoomed 5X using Fluoview software program Version 4.3. The positive and negative cells were read in the same fluorescence channel with the same settings.

\section{Results}

Secreted proteome of THP1 cells

We first tested the feasibility of isolating the secreted proteome of a pure population of cells utilizing THP1 [12], an established cell line of MO-myeloid lineage. THP1 cells were grown in serum-free medium for 7 days. The secreted proteins were separated by HPLC and then analyzed by either Edman degradation or by LC-tandem mass spectrometric analysis after trypsin digestion. The major proteins identified in the secreted proteome are lysozyme, chitinase 3-like 1 (YKL-40), proteinase 3, azurocidin, cathepsin G, beta-2 microglobulin, cystatin $\mathrm{C}$, desmoglein-1, secretory granule proteoglycan core protein, adenyl cyclase-associated protein (CAP1), junction plakoglobin, plakophilin1, moesin, palmitoyl-protein thioesterase, alpha-enolase, and prostaglandin-H2 D-isomerase (unpublished results). Azurocidin, and cathepsin $\mathrm{G}$ are known to have chemotactic activity [2]. Lysozyme and proteinase 3 are known to be involved in inflammation [2]. The secreted proteome analysis of THP1 resulted in identification of proteins functionally related to monocyte activity.

Secreted proteomes of MO/MA isolated from ascites of patients with EOC

Our secreted proteome study of the THP1 cell line demonstrated the feasibility of identifying a variety of functionally relevant proteins produced by a purified cell population. We utilized the same approach to analyze the secreted proteome of ascitic fluid MO/MA, which we isolated from patients with EOC using a Ficoll-Hypaque density cushion followed by adherence. Similarly, we 
Fig. 1 Alignment of 14-3-3 zeta with its other isoforms and the peptides identified by mass spectrometry analysis. Multiple sequence alignment was performed using the online alignment tool MAFFT (ver. 5.667). The peptides identified in secreted proteome analysis by mass spectrometry are highlighted either in boldface or underline. The peptides in boldface are sequences unique to $14-3-3$ zeta which were identified in the secretome analysis

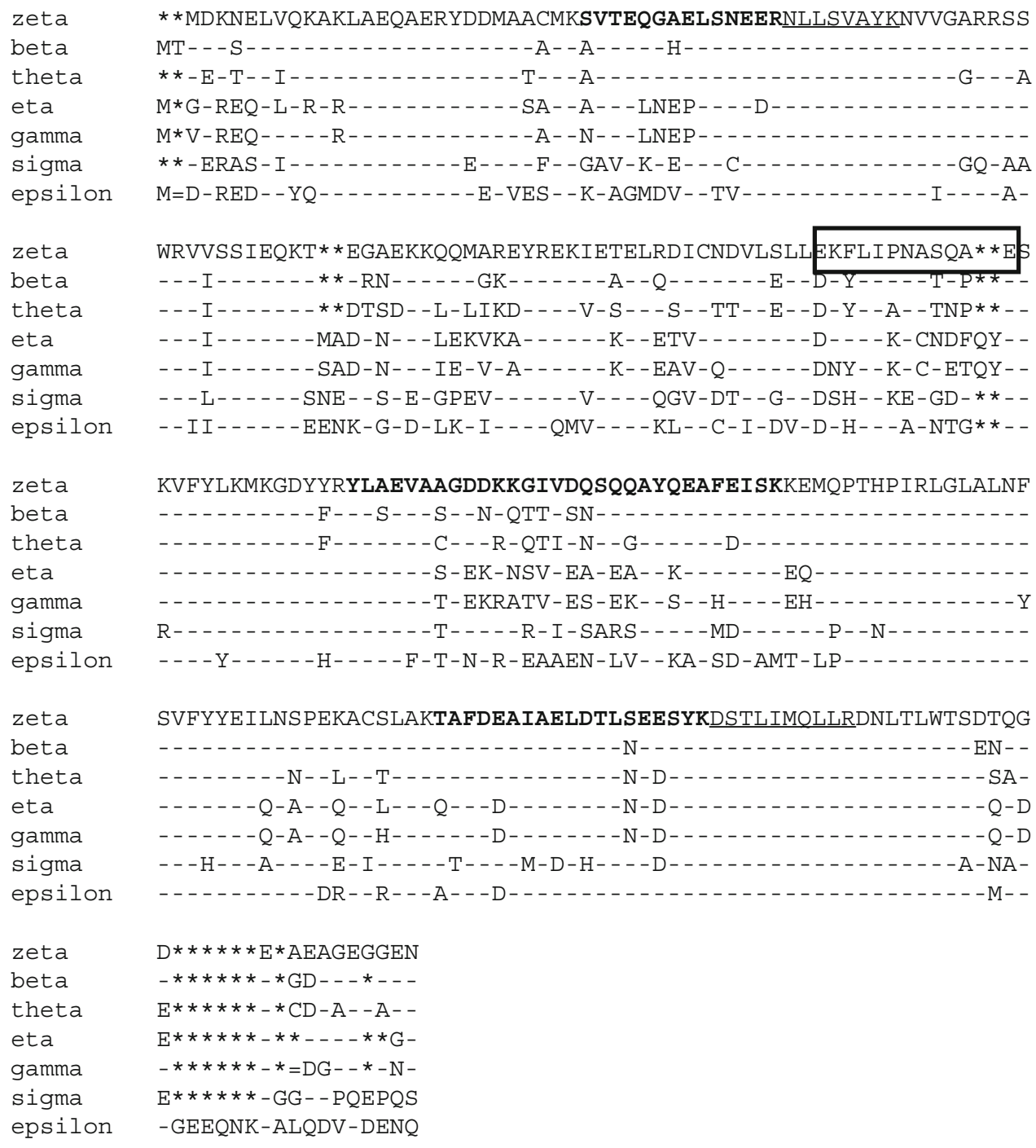

Peptides matched for mass spectrometric identification:

Patient\#290: SVTEQGAELSNEER, YLAEVAAGDDKK, NLLSVAYK, GIVDQSQQAYQEAFEISK, TAFDEAIAELDTLSEESYK, DSTLIMQLLR

Patient\#288: SVTEQGAELSNEER, YLAEVAAGDDKK

Patient\#287: SVTEQGAELSNEER

The peptide used to generate antibody is shown in box. analyzed the secreted proteome of buffy coat specimens, isolated using an MO isolation kit and MACS separator. Ascitic MO/MA were selected because they are the major representative of cell populations that contribute to the EOC inflammatory microenvironment and because of the facility with which they can be isolated and studied. MO/ MA isolated from ascitic fluid as adherent cells provided more than $90 \%$ purity and showed $99 \%$ viability. The purified MO/MA were grown in serum-free medium, either with or without MCSF. However, inclusion of MCSF in the media had no apparent influence on viability of the isolated
MO/MA. Non-adherent cells were removed from the cultures after 1-2 h. MO/MA supernatant were collected between 2 and 9 days from viable adherent MO/MA cultures. A suitable ascitic MO/MA control was unavailable from patients with benign disease, so column purified buffy-coat MO/MA isolated from normal donors were used for comparison (Table 1). Two hundred microliters of the ascitic MO/MA supernatants were separated by reversedphase HPLC, digested with trypsin, and analyzed by LCtandem mass spectrometry. Using this strategy, we identified 30-60 proteins from the supernatants of ascitic MO/ 
MA. For example, from one patient's sample we identified 53 total proteins that were divided into 4 groups: 36 proteins common to buffy coat; 16 proteins found only in one or two patients but not in all patients; and one common protein, 14-3-3 zeta, found in ascitic MO/MA supernatants from 3 of 3 EOC patients but not in supernatants of buffy coat cells (Table 1$)$.

The 14-3-3 zeta unique peptide SVTEQGAELSNEER (residues 28-41) was identified in all 3 patients. The zeta unique peptide YLAEVAAGDDKK (residues 128-139) was identified in patients \#290 and \#288. The zeta unique peptide GIVDQSQQAYQEAFEISK (residues 140-157) and TAFDEAIAELDTLSEESYK (residues 194-212) were also identified in patient \#290.

Further study was therefore focused on the 14-3-3 zeta protein. We also analyzed secreted proteins from CD163 + and CD163- cells using the MACS separator columns as described above. Secreted proteomes were analyzed in the same manner after 24 and $48 \mathrm{~h}$ incubations (Supplementary Table S1).

Immunoblot and mass spectrometric analysis of 14-3-3 zeta confirmed its secretion into the ascites of all patients with ovarian cancer

Ascitic fluid utilized in this study was obtained from 8 patients with advanced, untreated EOC of the most common histology, which is serous carcinoma stage 3 (5 patients) or stage 4 (3 patients). 14-3-3 zeta was identified in ascitic fluid from all 8 patients (Fig. 2). LC-MS/MS analysis of the gel bands corresponding to the bands in immunoblot analysis confirmed presence of 14-3-3 zeta protein by detecting peptides with sequences unique to the zeta isoform. The 14-3-3 zeta unique peptide SVTEQGAELSNEER (residues 28-41) was identified in all 8 samples. The zeta unique peptide GIVDQSQQAYQEA FEISK (residues 140-157) was also identified in samples 5, 6,7 , and 8 . The zeta unique peptide YLAEVAAGDDKK (residues 128-139) was identified in sample 6.

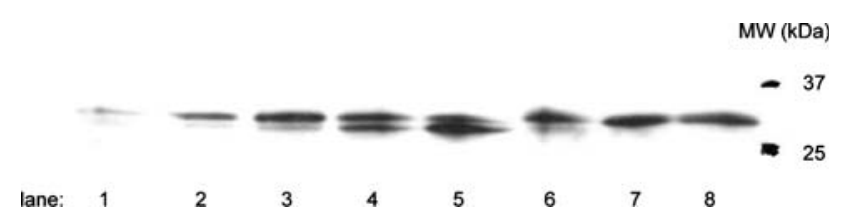

Fig. 2 Immunoblot and mass spectrometric analysis confirmed of 143-3 zeta in the ascites of 8 patients with EOC. The amount of ascitic fluid used to detect 14-3-3 zeta for immunoblot analysis was $2.5 \mu \mathrm{l}$ for lanes $1-4,1.3 \mu \mathrm{l}$ for lanes 5 and 6 , and $0.42 \mu \mathrm{l}$ for lanes 7 and 8 . All of the bands were confirmed by identifying peptides unique to the zeta isoform using LC-MS/MS analysis
Immunofluorescence studies

Since 14-3-3 zeta was first isolated from ascitic MO/MA, we stained a sample of unseparated ascitic cells and demonstrated costaining of CD163 + MO/MA for 14-3-3 zeta using the antibody we had developed with specificity for the 14-3-3 zeta analogue (Fig. 3a). Also seen are CD163- cells, of similar size and morphology to the CD163 + cells, which were also positive for 14-3-3 zeta. In contrast, large ascitic mesothelial cells $\left(\mathrm{CK}^{+}\right)$were negative or only weakly positive for 14-3-3 zeta. Approximately $50 \%$ of EOC associated MO/MA express the CD163 phenotype (1). Costaining of ascitic MO/MA for 14-3-3 zeta was also confirmed on a purified ascitic MO/MA (data not shown). In Fig. 3b, 14-3-3 zeta antibody staining of a cryostat section, obtained from a peritoneal biopsy from a patient with benign disease, primarily stains the peritoneal mesothelial $\left(\mathrm{CK}^{+}\right)$surface cells but not the resident CD163 + MO/MA. Further, specificity for the 14-3-3 zeta isoform on the surface mesothelial cells was confirmed by blocking the binding of the antibody with an epitope matching peptide.

We next utilized our antibody to describe 14-3-3 zeta's expression on EOC tumor and on peritoneal tissue specimens utilizing fluorescence costaining. We have previously shown that MO/MA represent a major leukocyte population in the peritoneum surrounding the tumor of EOC patients and there are large accumulations of MO/MA deep to the surface mesothelium [13]. Thirteen tumor specimens from representative EOC specimens were examined in detail (Fig. 4; Table 2). These experiments demonstrated that tumor cells $(\mathrm{CK}+)$, MO/MA $(\mathrm{CD} 163+)$, and endothelial cells $(\mathrm{CD} 31+)$, as well as other stromal cells, expressed the 14-3-3 zeta protein, though at differing frequency and intensity. Staining for 14-3-3 zeta protein was strongest on stromal cells and on MO/MA, particularly the cells found close to and within clumps of tumor cells in 10 of 13 samples. There was also positive costaining of CD31 endothelial cells for 14-3-3 zeta. Tumor cells that were positive for 14-3-3 zeta showed nuclear or cytoplasmic staining, and in 7 of 13 samples, cytoplasmic staining of tumor cells was negative, weak, or only focal (Table 2). We also found that 14-3-3 zeta was expressed on CD163 + MO/MA in peritoneum obtained from tumor free sites of 3 patients with EOC and on mixed Mullerian tumor of the ovary, but only focally on the CD3 + Tlymphocyte population (data not shown).

Co-culture experiment of 14-3-3 zeta with EOC cell lines

A recombinant FLAG-tagged 14-3-3 zeta was co-cultured with two EOC cell lines, $\mathrm{CaOV} 3$ positive cell line and 


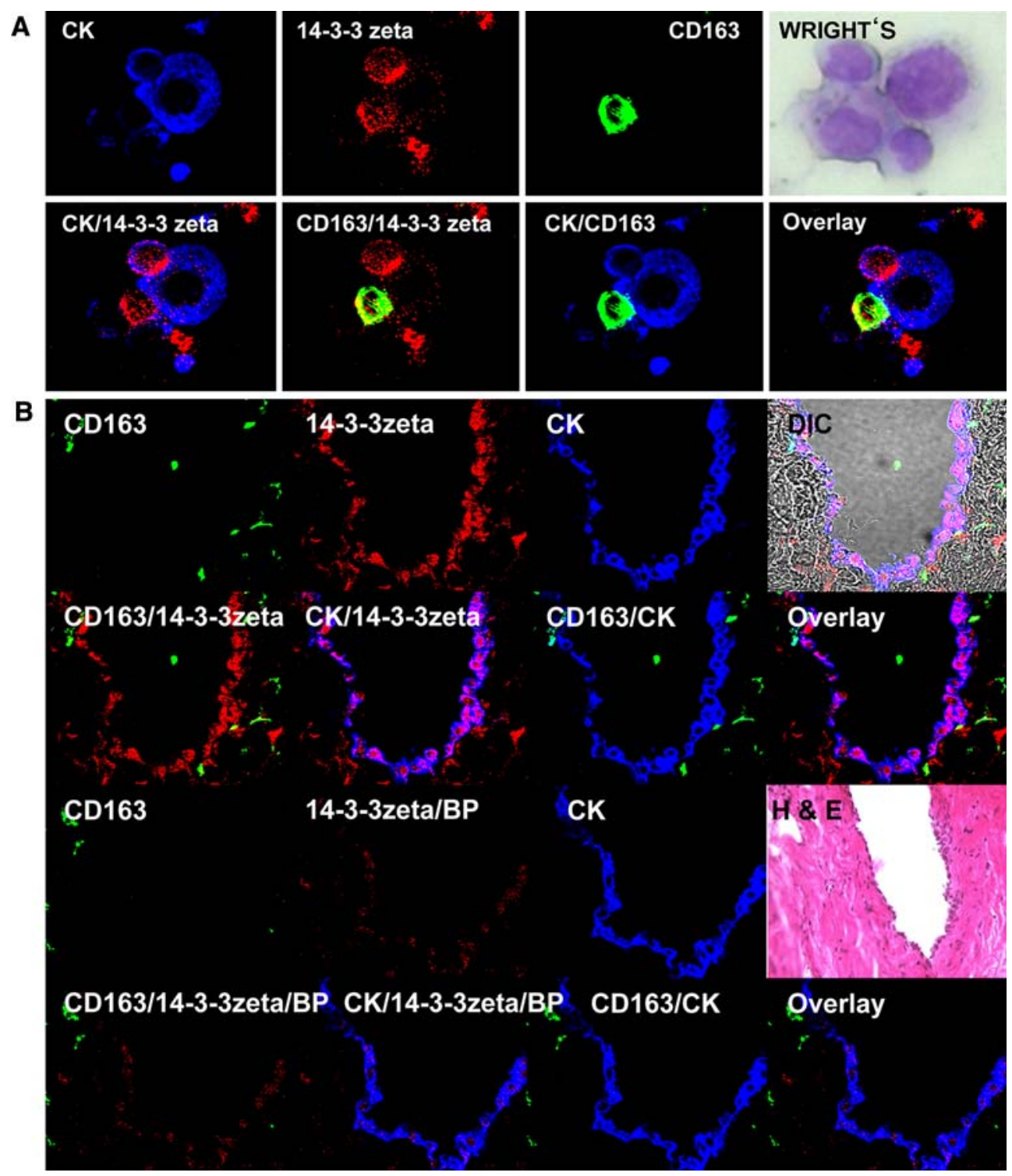

Fig. 3 a Immunofluorescence experiments revealed costaining of 143-3 zeta on CD163 + peritoneal MO/MA. Shown is triple indirect immunofluorescence costaining of cytospin preparation of ascitic peritoneal cells from ID294. Peritoneal cells were fixed with 4\% paraformaldehyde and stained with antibodies for the following antigens; cytokeratin $(\mathrm{CK})=$ blue, $14-3-3 \quad$ zeta $=$ red $\quad$ and CD163 = green reacted with Cy2, Cy3, and Cy5 conjugated affinipure antibodies corresponding to the isotype of primary antibody. Coexpression of 14-3-3 zeta on ascitic CD163 + cells is shown in yellow. The right upper panel shows Wright's stain of ascitic cells from the same sample (630 magnification). Images were made at $400 \times$ magnification and $4 \times$ zoom using Fluoview software version 4.3. b. Immunofluorescence staining of normal peritoneum for14-3-3 zeta identifies with surface mesothelium primarily. Expression of inflammatory MA cells marker CD163 (green), 14-3-3 zeta (red), and keratin/CK (blue) is shown on cryosections of benign peritoneum (ID283). Images from ID283 showed scant presence of CD163 + inflammatory cells. 14-3-3 staining is mainly identified with surface mesothelium. Specificity for 14-3-3 zeta expression was demonstrated by blocking with peptide, designated "BP." Red staining indicates the presence of 14-3-3 zeta. Top two panels lack blocking peptide. Lower two panels show absence or only weak staining of 14-3-3 zeta in presence of blocking peptide $(0.173 \mathrm{mM}$ peptide solution). The magenta staining reaction indicates costaining of mesothelial cells $(\mathrm{CK}+)$ with 14-3-3 zeta. H\&E and DIC images provide anatomic localization for the positively stained cells shown in laser confocal microscopy. Magnification for confocal is $400 \times$
MDA2774 cell line, to see whether the protein is taken up by EOC cells. In comparison to other EOC tumor lines tested these two cell lines did not have detectable 14-3-3 zeta by immunofluorescence (data not shown). Positive fluorescence specifically generated by anti-FLAG antibody were observed on CaOV3 positive cells and MDA2774 cells after 120 and 60 min incubations with FLAG-tagged 14-3-3-zeta, respectively (Fig. 5). 
Fig. 4 Immunfluorescence staining of tumor tissues from EOC patients. Costaining of cryosections from undifferentiated cancer, ID279 (upper panel), and endometrioid cancer, ID299 (lower panel), utilizing three antibody combinations reactive against a cytokeratin (CK) (blue)/14-33 zeta (red)/CD163 (green); b cytokeratin (green)/14-3-3 zeta (red)/CD31 (blue). Strong costaining of CD163 + MA, 14-3-3 zeta also strongly stains other cells in stroma but $\mathrm{CK}+$ tumor cells only focally. $\mathrm{H} \& \mathrm{E}$ preparations correspond to identical or similar structures shown in laser confocal microscopy panels. Results described in text $(400 \times$ magnification)

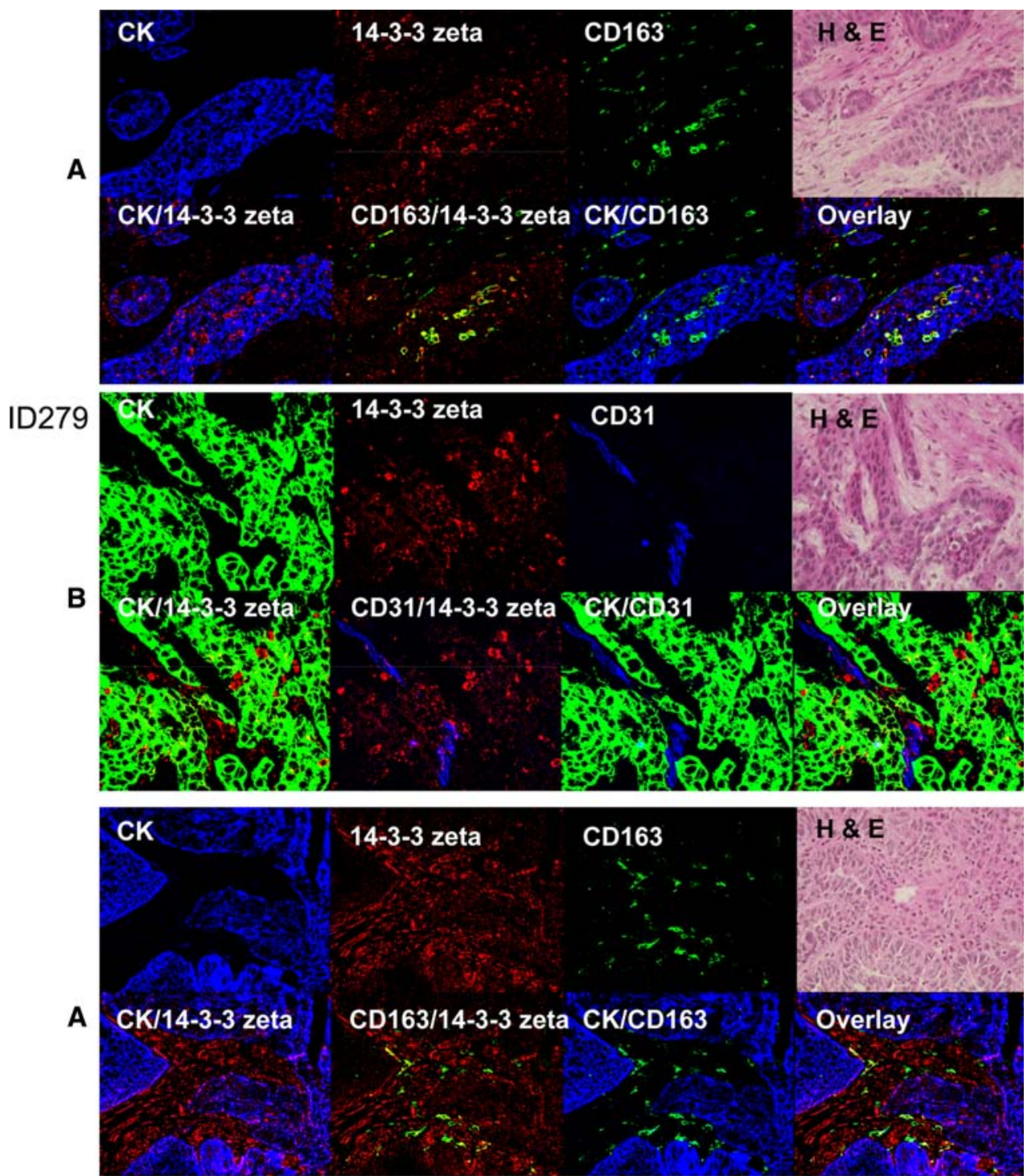

ID299

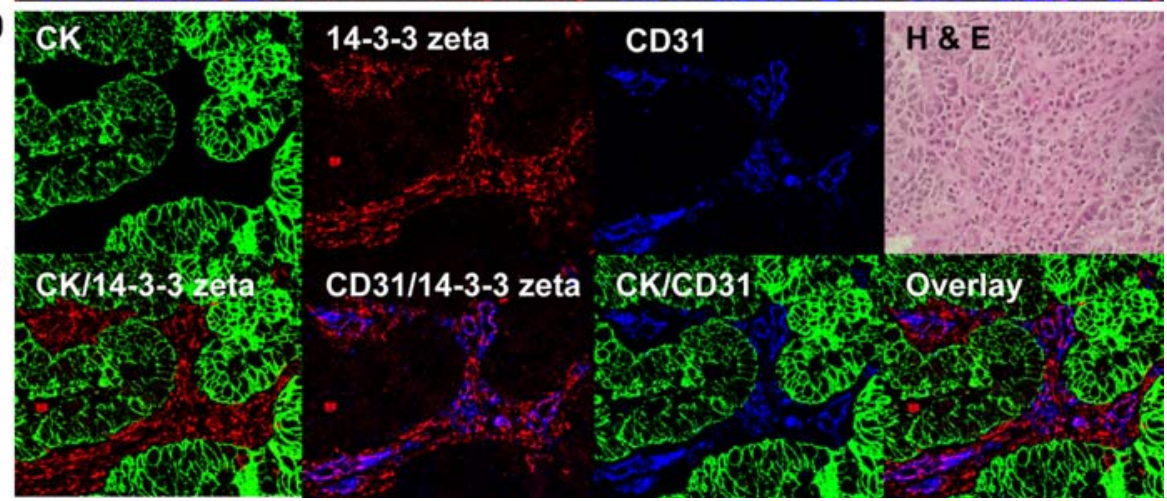

\section{Discussion}

In previous studies, we showed that the peritoneal tissues from EOC patients have an inflammatory profile that is consistent with MO/MA activation [14] by both differential microarray analysis [14] and by in situ cellular staining
$[4,13]$. In previous studies [5,9] we have also shown that ascitic MO/MA are inhibitory to $\mathrm{T}$ cell proliferation, or have lost the capacity to mediate opsonization and antibody-dependent cell-mediated cytoxicity [5], while they continue to produce cytokines and chemokines that could support the tumor. Although previous studies have 
Table 2 14-3-3 zeta localization on EOC and environmental cells of 13 patients

Quantitation: focal $(\mathrm{F})<5 \%$; weak (W) 6-33\%; moderate

(M) > 33-66\%; strong

(S) $>66 \%$

${ }^{\text {a }}$ Mullerian origin

b Stronger in proximity to tumor. $N T$ not tested

c 14-3-3 zeta isolated from MO/ MA supernatants of this patient

Fig. 5 14-3-3 zeta uptake by cutured EOC cells. Cell lines $\mathrm{CaOV} 3$ and MDA 2774 were cultured for $24 \mathrm{~h}$ at $5 \% \mathrm{CO}_{2}$, then treated with $2 \mu \mathrm{g}$ per well of FLAG-tagged 14-3-3 zeta or untreated cells as negative controls for different periods of time including 5, 30, 60 and $120 \mathrm{~min}$. CaOV3 positive cells are seen at $120 \mathrm{~min}$ incubation, while MDA2774 showed positive fluorescence at $60 \mathrm{~min}$ incubation of protein with FLAG-tag

\begin{tabular}{|c|c|c|c|c|c|c|c|}
\hline \multirow[t]{2}{*}{ ID\# } & \multirow[t]{2}{*}{ Histologic type } & \multirow{2}{*}{$\begin{array}{l}\text { Stage/ } \\
\text { grade }\end{array}$} & \multicolumn{2}{|c|}{ 14-3-3 丂/CK } & \multirow{2}{*}{$\begin{array}{l}14-3-3 \zeta / \\
\text { CD163 }\end{array}$} & \multirow[t]{2}{*}{ Stroma } & \multirow{2}{*}{$\begin{array}{l}14-3-3 \zeta \\
\text { CD31 }\end{array}$} \\
\hline & & & Nuclear & Cytoplasm & & & \\
\hline 243 & Mucinous & 3/Mod & $\mathrm{S}$ & $\mathrm{S}$ & W(scanty) & $\mathrm{S}$ & NT \\
\hline 256 & Endometrioid & 2/High & $\mathrm{W}$ & W & $S$ & $\mathrm{~S}$ & NT \\
\hline 279 & Undifferentiated $^{\mathrm{a}}$ & 4/High & Neg & $\mathrm{Neg}$ & $\mathrm{S}$ & $\mathrm{Neg}$ & M \\
\hline 287 & Serous $^{c}$ & 4/High & M & $\mathrm{Neg}$ & $\mathrm{W}^{\mathrm{b}}$ & $\mathrm{W}$ & NT \\
\hline 291 & Serous & 3/High & $\mathrm{S}$ & $\mathrm{S}$ & $\mathrm{W}^{\mathrm{b}}$ & M & NT \\
\hline 293 & Mixed Mullerian & 3/High & $\mathrm{Neg}$ & $\mathrm{F}$ & W & M & $\mathrm{S}$ \\
\hline 294 & Serous & 3/High & $\mathrm{S}$ & $\mathrm{S}$ & $\mathrm{F}$ (scanty) & M & NT \\
\hline 296 & $\begin{array}{l}\text { Serous and } \\
\text { endometrioid }\end{array}$ & 3/High & $\mathrm{F}$ & S & $\mathrm{M}^{\mathrm{b}}$ & S & NT \\
\hline 297 & $\begin{array}{l}\text { Serous and } \\
\text { endometrioid }\end{array}$ & 3/High & $\mathrm{F}$ & M & $\mathrm{W}^{\mathrm{b}}$ & $\mathrm{S}$ & NT \\
\hline 298 & Serous & 3/High & $\mathrm{Neg}$ & $\mathrm{Neg}$ & $\mathrm{M}^{\mathrm{b}}$ & $\mathrm{S}$ & NT \\
\hline 299 & Endometrioid & 3/High & Neg & $\mathrm{F}$ & $S^{b}$ & $\mathrm{~S}$ & $\mathrm{~S}$ \\
\hline 300 & Serous & 3/High & $\mathrm{S}$ & M & $S^{b}$ & $\mathrm{~S}$ & $S$ \\
\hline 301 & Serous & 4/High & Neg & $\mathrm{F}$ & $\mathrm{S}$ & $\mathrm{S}$ & NT \\
\hline
\end{tabular}

\section{CaOV3}

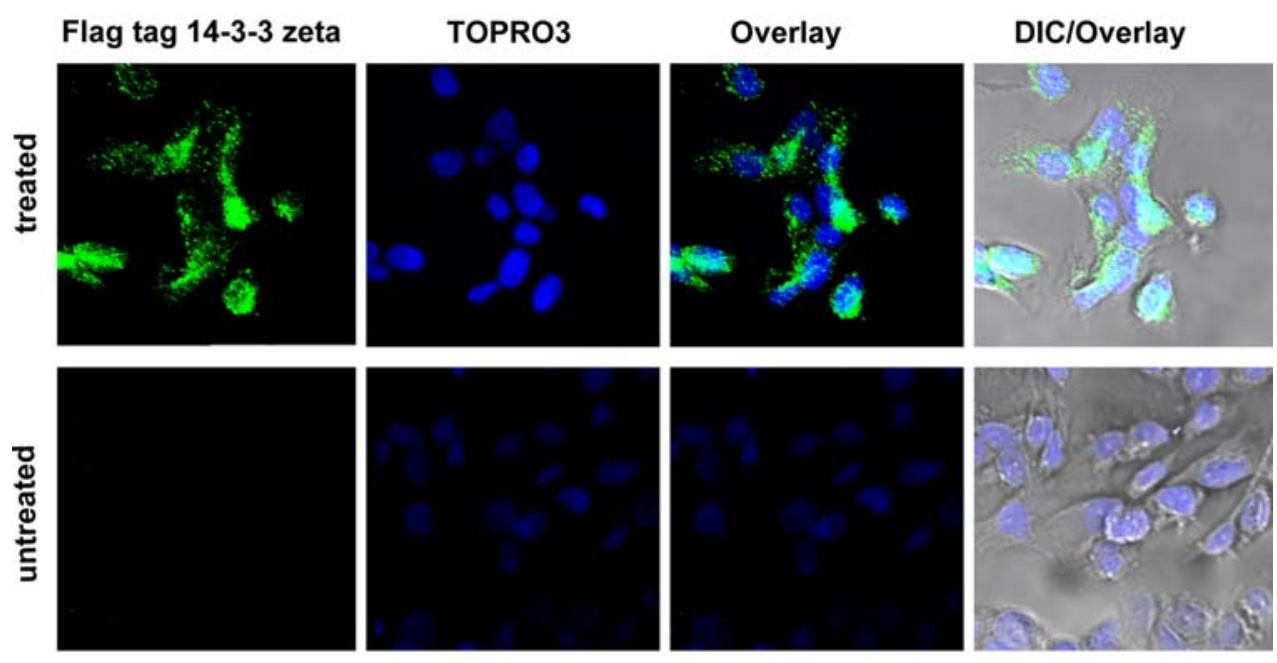

MDA 2774
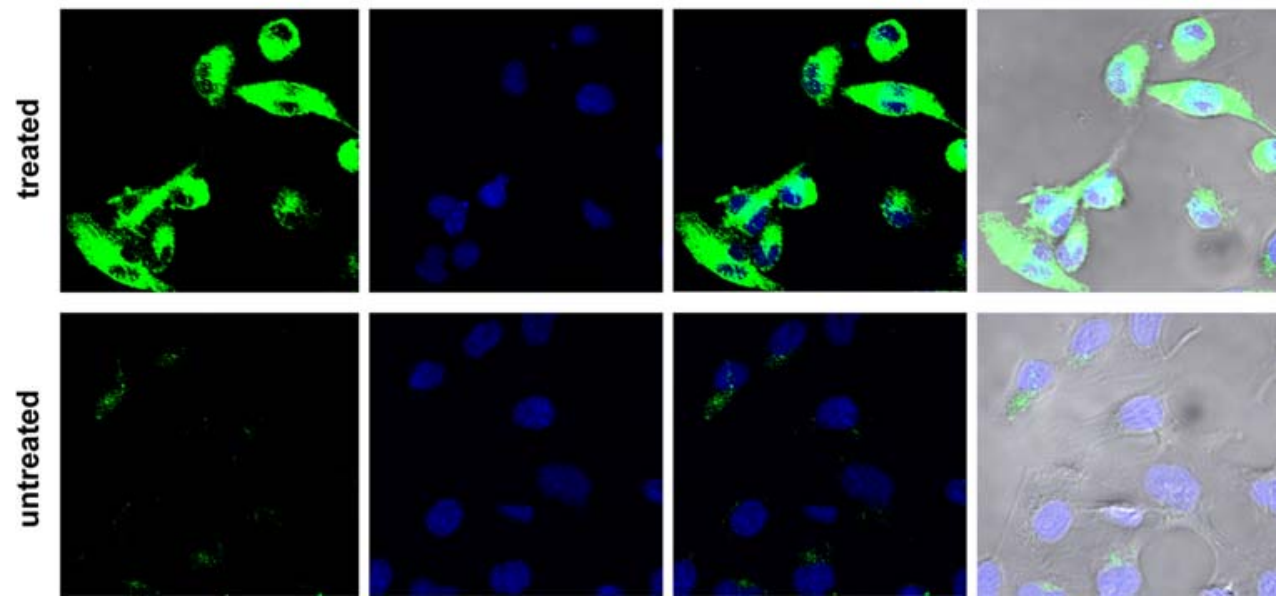
described a number of proteins secreted by MO/MA, they have generally employed antibody-based methods, using commercially available cytokine and chemokine detection kits. Here we employed an unbiased approach, utilizing mass spectrometry with the highest available sensitivity. Although not as sensitive as ELISA, it has enabled the discovery of a broad range of proteins secreted by a single population of cells. In particular, we were able to identify an adaptor protein, 14-3-3 zeta, which was commonly produced and secreted by three of three purified ascitic MO/MA samples. Furthermore, we produced a zeta specific antibody and identified 14-3-3 zeta in MO/MA that were detected in peritoneum and tumor of EOC patients. Other cells that expressed 14-3-3 zeta included tumor, either nuclear or cytoplasmic, stroma, and endothelial cells. 14-3-3 zeta was also identified in all eight of the EOC ascites specimens from chemotherapy-naïve patients. 14-33 zeta is also expressed in other normal cells such as the peritoneum as demonstrated in Fig. 3b. This is not surprising if it is considered that the peritoneum is an organ that as a protective function.

14-3-3 zeta is an important regulatory protein in intracellular signaling pathways and is known to interact with more than 100 cellular proteins, including oncogene and protooncogene products. Crystal structures have shown that 14-3-3 zeta forms a dimer and binds to proteins with tandem repeats of phosphoserine motifs [16]. It has been reported that 14-3-3 zeta blocks apoptosis by inhibiting the activation of p38 mitogen-activated protein kinase (MAPK) [15] and plays a critical role as an anti-apoptotic factor in cells. It is also reported that MAPK-activated protein kinase 2 (MAPKAPK2), a p38 MAPK-dependent inflammatory response mediator, regulates dimerization of 14-3-3 zeta through phosphorylation of Ser-58. In its phosphorylated form, 14-3-3 zeta appears unable to dimerize or bind to Raf-1 [10]. 14-3-3 zeta is also reported to interact with beta-catenin, enhance or inhibit beta-catenin-dependent transcription, facilitate activation of betacatenin through Akt, and possibly be involved in stem cell development [11]. Recently, it was reported that MO/MA infected in-vitro with human immunodeficiency virus type1 also secrete 14-3-3 zeta/delta [3].

Due to 14-3-3 zeta's many regulatory interactions, and since MAPK participates in LPS-induced proinflammatory cytokine production in macrophages and in other inflammatory responses its expression in ascites and ascitic cells was investigated. Since 14-3-3 zeta was the only protein identified in each of the ascitic MA supernatants, out of the 30-60 total proteins identified, but not in buffy coat MO/ MA supernatant it is reasonable to consider a possible role for this protein in the biology of EOC.

Macrophages release a number of inflammatory cytokines and chemokines. A speculated role of secreted 14-3-3 zeta in the tumor microenvironment might be either to enhance or inhibit tumor growth and proliferation. The data presented here represent the first description of the isolation and expression of this protein in cells from the EOC tumor environment. We also demonstrate for the first time that 14-3-3 zeta is secreted by ascitic MO/MA from EOC patients and present in malignant ascites of EOC patients. A functional role for 14-3-3 zeta as a secreted protein has not been elucidated; however 14-3-3 zeta has known diverse activities as an intracellular adaptor protein. We therefore conducted an experiment to determine whether the protein could be taken up by tumor cells that do not usually express easily detectable levels. In co-incubation experiments, utilizing 2 established ovarian tumor cell lines that do not express 14-3-3 zeta, we demonstrated that FLAG-tagged 14-3-3 zeta was indeed taken-up by EOC cells. The precise structure of 14-3-3 zeta isolated from ascites of EOC patients is unknown. However, the migration observed in SDS-PAGE during immunoblot analysis indicated that secreted form of the 14-3-3 zeta is intact. It is unproven that 14-3-3 zeta has a paracrine role in EOC. However, since the 14-3-3 zeta is an adaptor protein that is produced and secreted by tumor associated MO/MA at the tumor site, it is possible to speculate a possible role in regulating the inflammatory pathways of the EOC microenvironment. Since activated MO/MA are increased in the peritoneum of advanced ovarian cancer patients, it is also possible that 14-3-3 zeta might serve as a biomarker of tumor associated inflammation independent of its possible functions.

Acknowledgments We are grateful to Susan L. Davis for assistance with preparation of the manuscript and David Hawke for technical assistance.

Conflict of interest statement The authors have no financial conflict of interest.

Open Access This article is distributed under the terms of the Creative Commons Attribution Noncommercial License which permits any noncommercial use, distribution, and reproduction in any medium, provided the original author(s) and source are credited.

\section{References}

1. Chambers S, Kacinski B, Ivins C, Carcangiu M (1997) Overexpression of epithelial macrophage colony-stimulating factor (CSF-1) and CSF-1 receptor: a poor prognostic factor in epithelial ovarian cancer, contrasted with a protective effect of stromal CSF-1. Clin Cancer Res 3:999-1007

2. Chertov O, Yang D, Howard OM, Oppenheim JJ (2000) Leukocyte granule proteins mobilize innate host defenses and adaptive immune responses. Immunol Rev 177:68-78

3. Ciborowski P, Kadiu I, Rozek W, Smith L, Bernhardt K, Fladseth M, Ricardo- Dukelow M, Gendelman HE (2007) Investigating 
the human immunodeficiency virus type 1-infected monocytederived macrophage secretome. Virology 363:198-209

4. Freedman RS, Wang E, Voiculescu S, Patenia R, Bassett RL, Deavers M, Marincola FM, Yang P, Newman RA (2007) Comparative analysis of peritoneum and tumor eicosanoids and pathways in advanced ovarian cancer. Clin Cancer Res 13:57365744

5. Gordon IO, Freedman RS (2006) Defective antitumor function of monocyte-derived macrophages from epithelial ovarian cancer patients. Clin Cancer Res 12:1515-1524

6. Kassim SK, El-Salahy EM, Fayed ST, Helal SA, Helal T, Azzam ED, Khalifa A (2004) Vascular endothelial growth factor and interleukin- 8 are associated with poor prognosis in epithelial ovarian cancer patients. Clin Biochem 37:363-369

7. Lewis CE, Pollard JW (2006) Distinct role of macrophages in different tumor microenvironments. Cancer Res 66:605-612

8. Liu D, Bienkowska J, Petosa C, Collier RJ, Fu H, Liddington R (1995) Crystal structure of the zeta isoform of the 14-3-3 protein. Nature 376:191-194

9. Loercher AE, Nash MA, Kavanagh JJ, Platsoucas CD, Freedman RS (1999) Identification of an IL-10 producing HLA-DR-negative monocyte subset in the malignant ascites of patients with ovarian carcinoma that inhibits cytokine protein expression and proliferation of autologous T cells. J Immunol 163:6251-6260

10. Powell DW, Rane MJ, Joughin BA, Kalmukova R, Hong JH, Tidor B, Dean WL, Pierce WM, Klein JB, Yaffe MB, McLeish KR (2003) Proteomic identification of $14-3-3$ zeta as a mitogen- activated protein kinase-activated protein kinase 2 substrate: role in dimer formation and ligand binding. Mol Cell Biol 23:53765387

11. Tian Q, Feetham MC, Tao WA, He XC, Li L, Aebersold R, Hood L (2004) Proteomic analysis identifies that 14-3-3zeta interacts with beta-catenin and facilitates its activation by Akt. Proc Natl Acad Sci USA 101:15370-15375

12. Tsuchiya S, Yamabe M, Yamaguchi Y, Kobayashi Y, Konno T, Tada K (1980) Establishment and characterization of a human acute monocytic leukemia cell line (THP-1). Int J Cancer 26:171176

13. Wang X, Deavers M, Patenia R, Bassett RL, Mueller P, Ma Q, Wang E, Freedman RS (2006) Monocyte/macrophage and T-cell infiltrates in peritoneum of patients with ovarian cancer or benign pelvic disease. J Transl Med 4:30

14. Wang E, Ngalame Y, Panelli MC, Nguyen-Jackson H, Deavers M, Mueller P, Hu W, Savary C, Kobayashi R, Freedman RS, Marincola FM (2005) Peritoneal and sub-peritoneal stroma may facilitate regional spread of ovarian cancer. Clin Cancer Res 11:113-122

15. Xing H, Zhang S, Weinheimer C, Kovacs A, Muslin AJ (2000) 14-3-3 proteins block apoptosis and differentially regulate MAPK cascades. EMBO J 19:349-358

16. Yaffe MB, Rittinger K, Volinia S, Caron PR, Aitken A, Leffers H, Gamblin SJ, Smerdon SJ, Cantley LC (1997) The structural basis for 14-3-3: phosphopeptide binding specificity. Cell 91:961-971 